\title{
ACTIVATION ANALYSIS IN CLINICAL AND BIOLOGICAL RESEARCH
}

\author{
J. M. A. Lenihan \\ Department of Clinical Physics, University of Glasgow, Scotland
}

\begin{abstract}
Activation analysis, the first technique to allow the detection and accurate estimation of many trace elements in the human body, has been widely used in trace element studies, but important problems remain to be tackled before the biochemistry and physiology of the minor elements is adequately understood.

Topics of potential interest at the present time include the relationship of the chemical environment to disease, the analysis of the intact living body, the estimation of bulk elements and the study of dynamic metabolic processes. The limited success of activation analysis to date is attributable more to lack of imagination than to shortcomings in the technique.
\end{abstract}

This paper will not try to go over the ground that is well covered in numerous textbooks, monographs and reviews but will attempt a critical appraisal of the successes, shortcomings and continuing problems of activation analysis in medicine and biology.

Activation analysis is one of the many novel and sensitive analytical techniques developed in recent years ${ }^{1}$. It is particularly interesting because it was the first to achieve spectacular improvements in sensitivity-often by a factor of a thousand and sometimes by a factor of a million for particular elements. The arrival of activation analysis marked the beginning of the liberation of analytical chemistry from the unexciting task of counting atoms and opened up new opportunities for the analyst to contribute to the progress of medicine, science and industry - not only by making measurements (to provide answers to other people's questions) but by helping to formulate the questions.

\section{BASIC PRINCIPLES}

Activation analysis is a by-product of nuclear science and might be briefly described as instant isotopes. More precisely it is the revelation of atomic composition through the modification of nuclear behaviour. Most of the earlier methods of chemical analysis - and some of the more recent techniques - depend on the behaviour of electrons in the outer parts of the atom; it is of course these electrons which are responsible for most of the observable physical and chemical properties of materials.

Activation analysis depends on the properties of the nucleus. These 


\section{J. M. A. LENIHAN}

properties are not always obvious; although electrons are rather easily displaced or rearranged, the particles composing the nucleus are very tightly packed and do not respond to heat, electricity or chemical manipulation. For most elements, the simplest way to provoke the nucleus is to bombard the sample with thermal neutrons. Fast neutrons, other subatomic particles and high-energy radiations may also be used-but nuclear reactors, now quite widely available throughout the world, provide a cheap and convenient source of thermal neutrons.

The capture of a thermal neutron usually produces a radioactive isotope. The properties of the isotope, such as its half-life and the nature and energy of the radiations that it emits, are usually sufficiently specific to allow the confident identification of one or more elements in the irradiated sample. A quantitative estimation is usually made by comparison of the irradiated sample with a simultaneously irradiated standard containing a small amount of the element under investigation. Often the radioactivity of a particular element can be identified even in the presence of many other induced activities. When this is the case, gamma-ray spectroscopy of the irradiated sample and of the standard is enough for the required analysis. In order to obtain the ultimate sensitivity it is, however, usually necessary to resort to radiochemical separation of the desired activity from the irradiated sample.

Activation analysis has a number of obvious advantages. It is virtually free of errors due to impurities in the reagents and to contamination-so long as no contamination occurs before irradiation. The technique is also extremely sensitive (allowing estimation down to $10^{-12} \mathrm{~g}$ for some elements and $10^{-10} \mathrm{~g}$ for a great many more) and is quite specific, even for elements which are very difficult to distinguish in terms of the electronic properties used in traditional methods of analysis. Activation analysis has the further advantage that it can be non-destructive, allowing the recovery of the sample virtually unchanged or the repeated analysis of the same sample; these considerations are important in forensic science.

The principal disadvantage of activation analysis is that it requires access to a nuclear reactor or some other source of particles of irradiation. It is also, for some elements, rather slow, since the irradiation time may be a day or more. On the other hand, activation analysis lends itself to automated techniques or to batch processing, allowing 50 or 100 samples to be analysed in a comparatively short time.

There are three main applications of activation analysis. The first and most obvious is for the determination of trace elements or other minor constituents. This technique, which provided the first useful application of activation analysis - in the study of reactor materials during the 1939-45 war - has also been widely used in medicine and biology, though perhaps the results obtained have not so far been commensurate with the effort expended. Secondly, activation analysis is useful for the rapid estimation of bulk elements - for example, carbon and other constituents of coal and nitrogen in animal feeding stuffs. Thirdly, activation analysis has been applied to dynamic processes allowing, for example, the estimation of total body burdens of certain elements and, by measurements on the subject alone, the estimation of average daily dietary intakes of certain elements. 


\section{TRACE ELEMENTS-ESSENTIAL OR FORTUITOUS?}

The study of trace elements is a tantalizing problem which has been greatly advanced in recent years by the exploitation of activation analysis. The basis of the problem is that, of the 90 naturally occurring chemical elements, only about 25 are known with certainty to be essential to the life of man. Leaving aside the noble gases a few naturally radioactive or very toxic elements, there remain about 50. Many of these are found in the tissues of the body - usually at very low levels, though sometimes in surprisingly large amounts - but for most of them we have very little biochemical information. Until the arrival of activation analysis, it was impossible to obtain reliable estimates of the amounts of these minor elements present in different tissues.

This problem is now being tackled (by activation analysis and by more recently arrived techniques), but we are not yet very much further forward. The important question is to decide which of the minor elements are essential and which are fortuitous. There are a number of clues. If the concentration of a particular element in a particular tissue of the body varies widely between one individual and the next, it may be inferred that there is no physiological stabilizing mechanism and therefore that the element does not have an essential role. If (for example) we measure the concentration of sodium or manganese in any tissue of the body, we find a very narrow range. On the other hand, for elements such as lead, mercury and arsenic, the dispersion of the results is quite large.

These considerations are, however, not in themselves enough to distinguish between an essential and a non-essential element. To answer this question we need to demonstrate harmful effects resulting from a deficiency and from an excess of the element concerned. Since most of the minor elements are present in the body in total amounts of only a few milligrams, it is, for many of them, rather difficult to prepare diets with a sufficiently small content to produce deficiency symptoms.

After the intake levels corresponding to deficiency and excess have been identified, the next step is to identify the biochemical processes - and in particular the enzyme systems - in which the particular element is involved. This is a long process. At the turn of the century iodine was the only trace element known to be essential for the nutrition of man. In the succeeding century only four elements (copper, manganese, zinc and cobalt) were added to the list. Since then a further seven elements (molybdenum, selenium, chromium, tin, vanadium, fluorine and silicon) have been shown ${ }^{2}$ to be essential in animals, with a strong inference that they are essential also in man. Activation analysis was used in establishing the essential role of several of these elements.

\section{POISON AND POLLUTION}

One of the earliest medical uses of activation analysis was in the examination of hair samples for traces of metallic poisons. Arsenic and mercury (for example) seem, when once inside the hair, to stay indefinitely. Human hair, growing at the rate of $1 \mathrm{~cm}$ per month, provides a useful integrating dose- 


\section{J. M. A. LENIHAN}

meter for many pollutants, internal or external. By sectional analysis it is possible to estimate the time course of the internal contamination; this technique was successfully applied in Glasgow many years ago during the investigation of supposed poisoning of Napoleon ${ }^{3}$. By analysis of head hair and of body hair it is possible to obtain some discrimination between internal contamination (present in both samples) and external contamination (present only in head hair).

There are pitfalls in this technique, since several toilet preparations contain toxic metals - but the method has been usefully applied in the investigation of mercury hazards among dentists ${ }^{4}$. Finger nails and toe nails are also useful indicators of environmental contamination and, since they grow more quickly than hair, are suitable for examining short-term fluctuations.

Useful information is now being gathered about bodily contamination levels in times past. Hair samples of known date and origin are fairly readily available from the nineteenth century and, to a lesser extent, from earlier times. Though investigations of this kind are still at an early stage, there is evidence that our ancestors 100 or 200 years ago endured somewhat greater exposure to lead, mercury and arsenic than we do today-despite the common belief that the environment has become dirtier in recent years. The three elements mentioned were of course widely used in medicine until a comparatively short time ago.

Another problem in environmental health, very suitable for attack by activation analysis, arises from the increasing realization that many important afflictions, including heart disease and cancer, are strongly linked with environmental factors. The evidence here comes from epidemiological studies (for example, where neighbouring villages or communities very similar in genetic and social structure show marked differences in the prevalence of particular diseases) and from studies on migrant populationswhich often take with them the morbidity pattern characteristics of their original homes, but after a relatively short time take on the morbidity pattern of the country to which they have moved.

In seeking the environmental factors responsible for such differences, it is reasonable to give close attention to the microchemical environment. Analysis of food, soil, cosmetics and hair and nail samples for a number of trace elements can be readily achieved at good sensitivity by activation analysis. Work of this kind is going on in a number of laboratories in research programmes organized or supported by the World Health Organization.

The analysis of tissue samples from living or dead subjects is a longestablished procedure which has in recent years become considerably extended through the exploitation of activation analysis. It is reasonable to suppose that knowledge of the chemical composition of the intact living subject - or of particular organs in the body-would sometimes be more useful than the study of isolated fragments of tissue.

\section{THE ANALYSIS OF MAN}

When we try to assess the problem of analysing the intact living body, we face four major difficulties. Firstly there is the problem of sampling. The body is by no means homogeneous; indeed some of its activities depend 
very much on localized concentrations of particular elements. Consequently we cannot reduce the complexity of the problem by analysing only a portion of the specimen. Secondly, the amount of a particular substance in the body does not necessarily correspond to its functional importance. At any given moment, the body contains quite a lot of material which has served its purpose and is proceeding towards elimination, as well as other materials which are still being prepared for their useful function. Thirdly, it must be admitted that biochemistry and physiology have not until now been greatly advanced by the study of the intact living subject. The body is a symposium of interconnected systems, and, in the chemical sense, seldom behaves as a single entity. Fourthly, we face a serious psychological obstacle. The analyst has for many years - in response to the demands of his clientsbecome accustomed to measuring very small amounts of material in very small samples.

If the analyst can overcome his obsession for small samples, he finds, not surprisingly, that his techniques and instruments are not always well adapted to the study of large samples, particularly if the sample is a living subject. The analyst usually prefers to work with ions rather than with atoms and molecules. Consequently he likes to have his samples in solution and to choose the solvent himself. If this is impossible, he usually tries to mobilize the ions in the sample with the aid of a flame, an arc or a spark.

In principle, it would be possible to measure the composition of a dead body by the conventional methods of wet chemistry, but the technical difficulties associated with a sample of mass $70 \mathrm{~kg}$ are enormous, even for the estimation of major elements. In attempting to estimate the minor elements by conventional methods, the difficulties are so serious that accurate analysis is virtually impossible.

\section{ACTIVATION IN VIVO}

Activation analysis certainly offers a theoretical possibility of escape from this impasse ${ }^{5}$. One method is to make the subject radioactive and study the induced activity. The application of this technique to an intact human subject involves considerable difficulty. Thermal neutrons, which are capable of producing measurable levels of induced activity, are strongly absorbed in the body. On the other hand, fast neutrons (which penetrate the tissues more easily) do not induce enough activity for accurate measurement if the radiation dose is to be kept within acceptable limits.

The solution usually adopted is to irradiate the subject with fast neutrons and to rely on collisions with hydrogen atoms in the body for thermalization. The body is not at all well designed for this experiment, since some important parts-for example, the feet, the forearms and the skull-contain bone covered with very little soft tissue.

One way out of the difficulty is to immerse the limbs in lucite tanks filled with water and to surround the skull with lucite. Even so, the variation in induced radioactivity per gram of bone may be as much as 25 per cent between the spine and the ankle.

Attempts have nevertheless been made to estimate total body calcium by neutron irradiation in vivo, followed by radioactive assay in a whole-body 


\section{J. M. A. LENIHAN}

counter. By this technique, it is certainly possible to produce, in a gamma-ray spectrometer, a visible peak corresponding to ${ }^{49} \mathrm{Ca}$. To translate this peak into the total mass of calcium in the body is, however, much more difficult, since there is at present no combination of equipment in the world fulfilling the two essential conditions for accuracy-uniform thermal neutron flux throughout the body and uniform sensitivity of detection, regardless of position in the body. Consequently it remains difficult to distinguish between a genuine difference in total body calcium and a redistribution of calcium within the body. Furthermore the conversion ratio between ${ }^{49} \mathrm{Ca}$ counts and total body calcium varies according to the size and shape of the patient and cannot be standardized. The same considerations restrict the usefulness of activation analysis in estimating the total body content of other elements.

\section{THYROID IODINE}

Activation analysis of limited regions of the body presents less formidable problems. The thyroid gland is an obvious target for work of this kind, since it contains a substantial part of the body's total iodine. In principle, the irradiation of the thyroid with thermal neutrons will produce detectable amounts of ${ }^{128} \mathrm{I}$, but formidable difficulties arise in attempts to standardize the measurements - that is, to relate ${ }^{128} \mathrm{I}$ counts to total thyroid iodine.

The main problem is that the size, shape and position of thyroid and the distribution of iodine within the gland are never accurately known. On the other hand, because of the small amount of ${ }^{128} \mathrm{I}$ produced by an acceptable dose of neutrons (bearing in mind the radiation hazard) it is necessary to have the scintillation detectors very close to the neck during the assay of induced activity; for this reason the geometrical uncertainties just mentioned are more serious.

These difficulties can be overcome by the use of ${ }^{129} \mathrm{I}$ as an internal standard-a technique ${ }^{6}$ successfully used in the estimation of thyroid iodine in sheep but not so far applied to the human subject, because of reservations about the radiation dose. Work on the estimation of thyroid iodine by direct irradiation of the neck with reactor neutrons, and subsequent scintillation counting without an internal standard, has been in progress since 1967. The meagre results so far published ${ }^{7}$ are not encouraging.

\section{PROMPT GAMMA-RADIATION TECHNIQUES}

Although thermal neutrons are usually preferred for in vivo activation, there are interesting possibilities (a) involving fast neutrons and (b) using the prompt gamma-radiation emitted immediately after the incorporation of the bombarding thermal neutron into the target nucleus. The more usual method of measuring induced radioactivity involves study of the delayed gamma-radiation emitted in the radioactive decay of the newly formed compound nucleus. The gamma-radiation emitted immediately after neutron capture is, however, often quite abundant and is quite specific (in terms of energy) of the elements contributing to it. The radiation detectors must be carefully shielded from the neutron beam. If a cyclotron is used as a neutron source, the gamma-ray background from the target is troublesome 
and it is usually necessary to deliver the neutrons in a succession of short pulses, counting the prompt gamma-radiation during the intervals between pulses.

Total body hydrogen ${ }^{8}$ and nitrogen ${ }^{9}$ have been estimated by measurement of prompt gamma-radiation after neutron activation analysis. There are attractive possibilities also in the use of pulsed sources of neutrons or highenergy $x$-radiation to produce isomeric states with half-lives in the millisecond or microsecond region. Cross-sections for reactions of this kind are often very large, allowing adequate sensitivity with small radiation doses.

Elsewhere in the biological realm, fast neutron activation has been used for the estimation of nitrogen in foodstuffs; this measurement is significant because of its close relationship to protein content. As long ago as 1967, commercially available equipment allowed the estimation of nitrogen in grain products with reproducibility of one per cent at a cost of $\$ 1.80$ per sample. On this basis, the technique is comparable in cost and precision with the familiar Kjeldahl process. Industrial uses of neutron activation analysis have, however, been very slow to develop.

The applications of activation analysis so far discussed have all been related to human or animal subjects. A recent growth of interest in environmental problems suggests useful possibilities of activation analysis of plant tissuesfor example, in relation to the uptake of nutrients and essential trace elements from the soil ${ }^{10}$.

\section{DYNAMIC STUDY}

Most of the medical and biological applications of activation analysisand indeed of other sensitive analytical techniques - are concerned with static systems such as tissue samples. A few years ago my colleagues Orr and Gillespie showed ${ }^{11}$ that a considerable bonus of useful information could be obtained by exploiting the fact that the chemical constituents of a living subject are always in movement. Food is ingested and subjected to various metabolic changes; some of the products are incorporated in the body tissues and others are excreted. In a normal healthy subject, the rate of ingestion of any element is equal to the rate of excretion. These apparently trivial observations add a new dimension to the repertoire of the analyst.

To see how the method is used, consider what happens when a small amount of a tracer substance (usually but not necessarily radioactive) is introduced into a system of the body. The amount present in (for example) the blood will rise very quickly after intravenous injection and more slowly after oral administration, reaching a maximum and then declining. The retention curve usually takes the shape of a single exponential slope before long - though in some instances (for example, when considering calcium in bone) the existence of more than one compartment may be evident for rather a long time.

When the single exponential slope has been reached, the area under the curve can be extrapolated to infinite time. This quantity we call the occupancy of the system for the substance being simulated by the tracer; in certain circumstances it is equal to the average residence time of the substance in the system. 
We now define the capacity of the system as the total amount of the substance in question that it contains. It is rather easily shown that (under certain conditions which are usually fulfilled) the ratio of capacity to occupancy is equal to the average rate of intake of the substance; if (as is usually convenient) the occupancy is measured in days, the ratio will be the average daily intake.

\section{THE OCCUPANCY PRINCIPLE}

Where the occupancy and the capacity can both be measured-for example, in the blood the Occupancy Principle gives a simple method of estimating the average daily dietary intake without the necessity to examine the diet, since all the measurements can be made on the subject or on samples taken from him; it is of course necessary that the subject should be in metabolic equilibrium for the substance in question over the period of the experiment.

In the form just mentioned, the Occupancy Principle is not usually convenient for application to the whole body, since the capacity (that is, the total amount of a particular substance in the body) is usually the unknown element in the metabolic equation.

However, a simple extension of the Occupancy Principle becomes very useful when we can find two interlinked systems-such as blood and whole body or thyroid gland and blood - such that the substance in question passes through one system in order to reach the other. In this situation the ratio of capacity to occupancy will be the same for each of the two systems.

Often we can measure without much difficulty the capacity and the occupancy for the blood and, by the use of a whole-body counter, we can measure the total body occupancy. This means that three of the four quantities in the equation are known and the fourth - the total body capacity - can be calculated. This technique has already been used for the estimation of total body bromine in man ${ }^{12}$ and total body selenium in the rat $^{13}$; the same method has been used to estimate total intrathyroid iodine in man $^{14}$. It is undoubtedly capable of further extension-a possibility which we are now pursuing in Glasgow in relation to various toxic metals.

There are other useful applications of the principle. If, for example, we measure the occupancy twice-once after oral administration and once after intravenous ingestion - the ratio of the two results gives the percentage absorption.

In order to use the Occupancy Principle, it is of course necessary to find a suitable isotope and establish that, after a time which is not too long in relation to the half-life of this isotope, the retention curve has reached its final exponential slope. These conditions are not fulfilled for every element but, in some instances, it seems likely that the Occupancy Principle will give the same information as could be obtained by activation analysis in vivo, but without the hazards of neutron dosage to the patient and without the necessity for elaborate equipment such as a neutron generator or a reactor adapted to the irradiation of human subjects.

\section{THE FUTURE}

What is the future of activation analysis? The late Richard Ogborn, a 
notable American pioneer of the technique, said many years ago that the applications are limited only by the imagination of the experimenter. This is a rather severe limitation. At times during the last 30 years, activation analysis has looked like an answer in search of a question. The capacity of the human mind for original thinking is severely limited. Consequently analysis has to a large extent been used to refine experiments already done or (but for the limitation of sensitivity) capable of being done by other techniques.

It is important also to recognize some of the limitations of activation analysis. In general, it is a technique for the detection of elements and does not help us much in identifying the chemical form in which the element occurs; this difficulty can sometines be overcome by combining activation analysis with other techniques such as thin-layer chromatography.

The possibilities of activation analysis have sometimes been overestimated. Automated multi-element analytical projects have been developed but not widely used, mainly because few organizations can provide enough samples to keep a system of this kind economically employed. Painstaking efforts to devise diagnostic tests for cancer (based on differences in trace element concentrations in blood or other tissues) are still being made, but have not proved useful; the differences between normal and malignant tissues are almost certainly too complex to be revealed by simple chemical analysis. Identification of individuals by trace element analysis of hair has proved more difficult than was expected and is still in the limbo of promising but unproved techniques.

To some extent activation analysis has been the victim of its own success. Many of the highly sensitive techniques now available to the analyst (for example, anodic stripping voltametry and atomic absorption) use no scientific principles that were not known many years ago. These techniques would probably not have been developed but for the achievements of the early activation analysts in breaking through the microgram barrier which for many elements marked the limit of conventional techniques.

After the initial breakthrough the distinctive contribution of activation analysis has been in encouraging the development of multidisciplinary teams including physicists, engineers, biologists and clinicians as well as chemists. In this process the analyst has been led out of the back room to join in the attack on some of the major problems of health and the environment which so greatly (and so rightly) excite the concern of mankind in the world of today.

\section{REFERENCES}

${ }^{1}$ For general accounts of the principles and applications of activation analysis see (for example): (a) Modern Trends in Activation Analysis (2 volumes), National Bureau of Standards Special Publication 312, Washington, DC (1969). Proceedings of the Third International Conference on Modern Trends in Activation Analysis, 1968.

(b) K. Kruger, Principles of Activation Analysis. Wiley: New York (1971).

(c) J. Radioanal. Chem. 15 (2) and 16 (1973). Proceedings of the Fourth International Conference on Modern Trends in Activation Analysis, 1972.

For the medical and biological applications, see:

(a) Nuclear Activation Techniques in the Life Sciences, IAEA : Vienna (1972) (also an earlier volume with the same title, 1967). 


\section{J. M. A. LENIHAN}

(b) D. Comar in Advances in Activation Analysis, Vol. 1, p 163. Academic Press: London (1969).

2 K. Schwartz, in Nuclear Activation Techniques in the Life Sciences. IAEA: Vienna (1972).

3 S. Forshufvud, H. Smith and A. Wassen, Archiv. Toxikol. 20, 210 (1964).

4 J. M. A. Lenihan, H. Smith and W. Harvey, Brit. Dent. J. 135, 365 (1973).

5 In vivo Neutron Activation Analysis. IAEA: Vienna (1973). A generally optimistic view of body composition studies.

6 J. M. A. Lenihan, D. Comar, R. Riviere and C. Kellershohn, J. Nucl. Med. 9, 110 (1968).

7 K. Boddy, R. M. Harden and W. D. Alexander, J. Clin. Endocrinol. Metab. 28, 294 (1964).

8 B. Carlmark and P. Reizenstein, in reference 5, p 113.

9 H. E. Palmer and W. B. Nelp, in reference 5, p 127.

10 G. J. Peterson, in Nuclear Activation Techniques in the Life Sciences, p 323. IAEA: Vienna (1972).

11 J. S. Orr and F. C. Gillespie, Science, 162, 138 (1969).

12 F. C. Gillespie, J. Shimmins and J. M. A. Lenihan, Radiochem. Radioanal. Letters, 4, 43 (1970).

13 B. Maziere, M. Maziere and D. Comar, in Nuclear Activation Techniques in the Life Sciences, p 359. IAEA : Vienna (1972).

14 R. Riviere, D. Comar, C. Kellershohn, J. S. Orr, F. C. Gillespie and J. M. A. Lenihan, Lancet, i, 389 (1969). 